\title{
SYSTEM CONCEPT DEVELOPMENT WITH VIRTUAL PROTOTYPING
}

\author{
James C. Schaaf, Jr. \\ Faye Lynn Thompson \\ BDM International \\ 950 Explorer Blvd. \\ Huntsville, Alabama 35806, U.S.A.
}

\begin{abstract}
Virtual prototyping with 3D drawing programs provides a means of rapidly developing system concepts and analyzing them for form, fit, logistics, human factors integration, and general feasibility analysis. The resulting models can be studied, viewed from different angles, and even "entered" by multidisciplinary design teams working in an integrated product team environment. The authors were involved in two separate system configuration developments that benefited from an initial virtual prototyping approach. The first example presents the development of an electric gun concept for a ballistic missile defense application. The gun model was based on current technology developments and experiment results, and was constructed to answer the question "what configuration can be assembled in the near term that would be air transportable?" The second example illustrates the development of a small waste remediation facility, and was constructed to provide a configuration baseline for study. The process of converting each project's miscellaneous available technical data into a correct high-fidelity model is presented, and key technical insights provided by the virtual prototype are also presented.
\end{abstract}

\section{INTRODUCTION}

Virtual prototyping is an aspect of information technology that permits analysts to examine, manipulate, and test the form, fit, motion, logistics, and human factors of conceptual designs on a computer monitor. It facilitates communication between different engineering disciplines during the early design process, and also provides quality illustrations that help "sell" the design or program. Garcia, et. al. state that the Department of Defense (DoD) defines a virtual prototype as "A computer-based simulation of a system or subsystem with a degree of functional realism comparable to a physical prototype" and virtual prototyping as "The process of using a virtual prototype, in lieu of a physical prototype, for test and evaluation of specific characteristics of a candidate design (Garcia, Gocke, and Johnson 1993)." Virtual prototyping can replace the expensive physical mockups constructed to test designs. In fact, there is a trend to skip the mockup phase as experience drives more sophisticated use of virtual prototypes during the design analysis process (Piplani, Mercer, and Roup 1994). The value of virtual prototyping is rapidly being recognized for a wide range of engineering applications. These applications range from illustrating the potential of a system early in the technology assessment activity or early developmental phase to detailed analysis of mature designs in the advanced engineering phase. Some of the uses include design form, fit, and function, logistics and human factors analysis. Perusal of trade magazines will show a growing proliferation of $3 D$ programs ranging from low cost $3 \mathrm{D}$ drawing applications to high cost $3 \mathrm{D}$ rendering of computer aided design (CAD) programs (Potter 1996, Murie 1996). Low cost drawing programs provide adequate accuracy and detail to support analysis during concept development (Schaaf, Thompson, and Churchwell 1994), while CAD is required for detailed engineering development of a maturing design.

\section{MODEL CONSTRUCTION}

Virtual prototypes are developed from concept drawings or blueprints, and contain as much detail as required to support the immediate need. The same model can be expanded later as more detail is required. For example, testing the fit of a proposed weapon system load on a vehicle may initially require only the length, width, and general shape of the frame. Later, as more details such as outriggers, hydraulic tanks, etc., are required; these can be added along with other underbody details to test for available space and interference. In the computer, the various parts are developed to scale and then are 
assembled the same way they would be on a fabrication shop floor. This allows the analyst to test assembly procedures and human factors as well as the form and fit of the final product. It also allows parts to be removed to show otherwise hidden components. Figure 1 illustrates subassemblies created for the construction of a military High Mobility Multipurpose Wheeled Vehicle (HMMWV) model.

Figure 2 illustrates a potential danger or abuse of the ease of using 3D drawings to create virtual prototypes and shows a large vehicle that appears feasible - yet no real engineering has been accomplished beyond the third axle. The front portion of this creation is based on an accurate model developed from drawings of a Stewart \& Stevenson candidate for the Army's new family of Medium Tactical Vehicles (MTV) (Stewart \& Stevenson 1993). All of the structure beyond the third axle is conjecture meeting the demands of the grandson of one of the authors. The model appears realistic and may be feasible, but it is not backed up with engineering projections for load bearing features. Taking such liberties during the projection of new technology into concept systems would be unethical and just poor engineering analysis. To maintain credibility, virtual prototypes at all stages of engineering development must be based on disciplined analysis and sound technology projections. Construction details must be backed up with engineering analysis to provide value and credibility. Useful models cannot be "fabricated" from materials such as Impossibillium and Unobtainium that

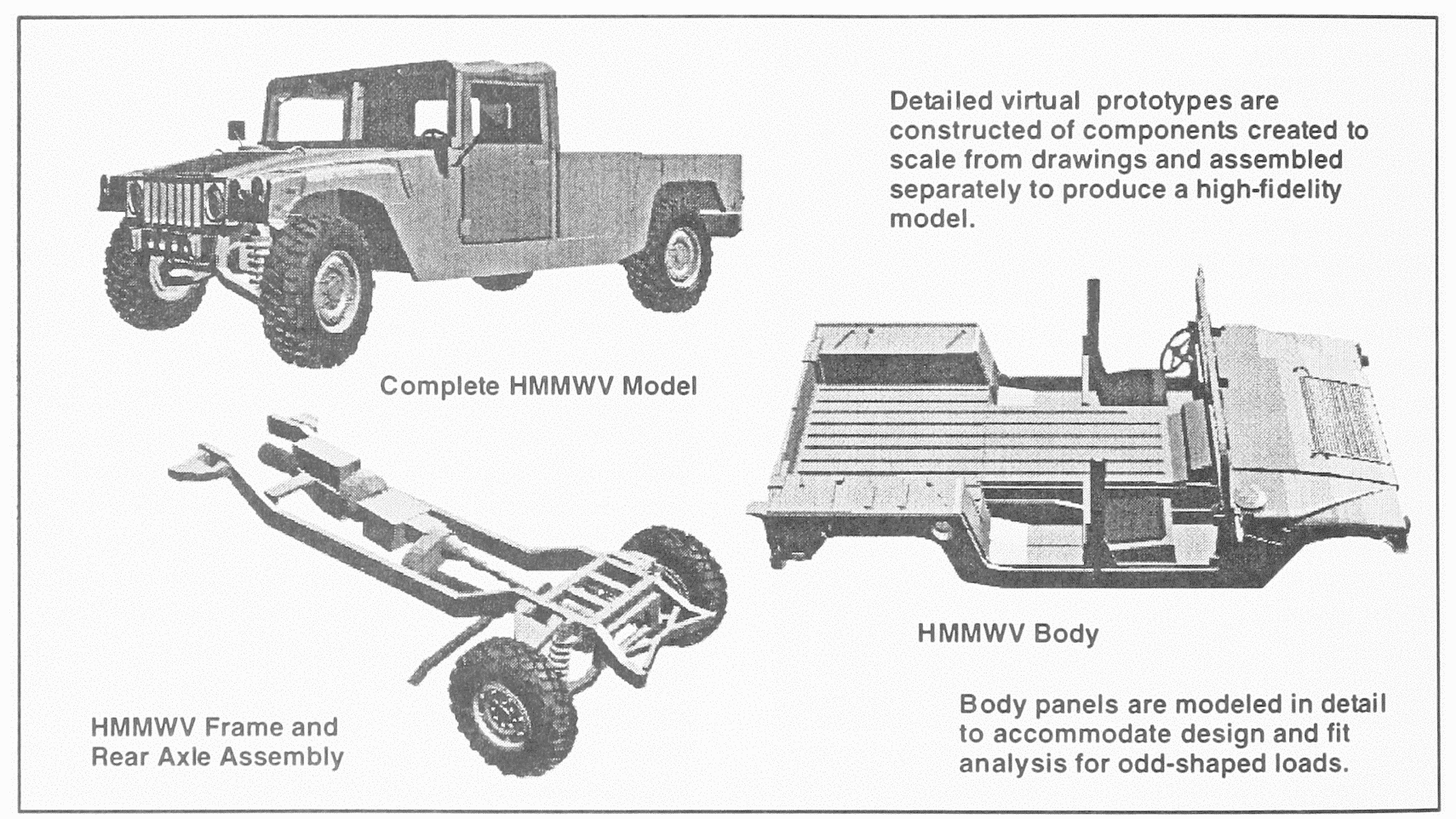

Figure 1: Military HMMWV Subassemblies

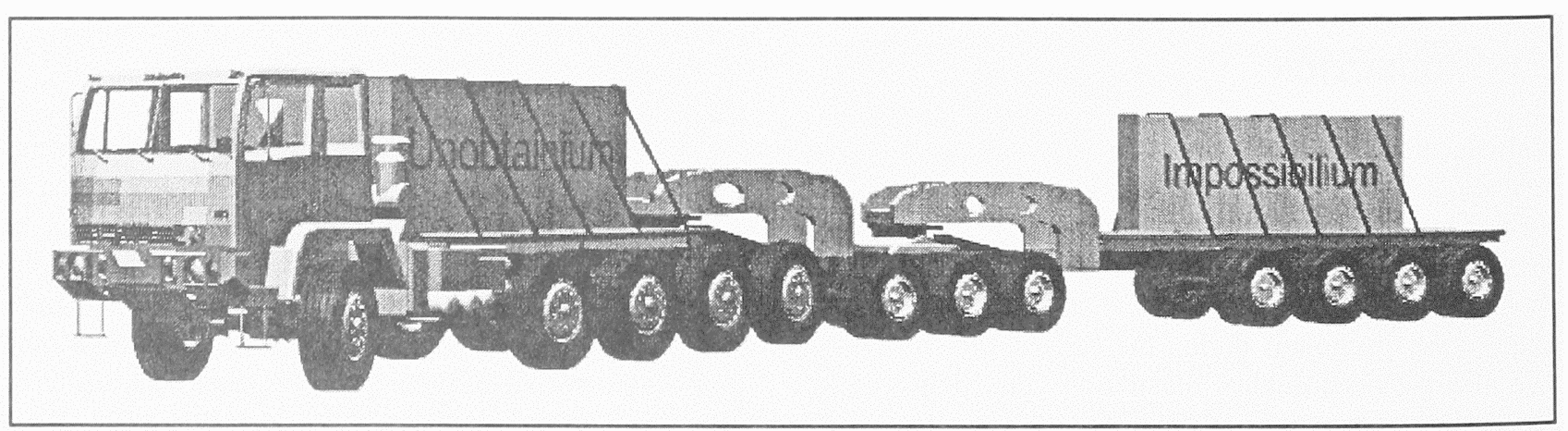

Figure 2: Heavy Hauler "Fantasy" 
produce a model that bears no realistic comparison to real world strength, performance, size, and weight.

\section{VIRTUAL PROTOTYPING IN THE EARLY CONCEPT PHASE}

The authors work primarily in the technology assessment and advanced concept phases where we are required to project new technology into system applications, and provide early answers relating to system size, fit, tactical feasibility, and transportability. At this early stage, system details are not fully developed nor even known, yet certain questions must be answered such as, can it be packaged in a tactical configuration?, how much will it weigh?, and can it be made C-130 transportable? These questions are particularly important when considering the requirements and constraints of defense systems, and are used in arguments supporting program continuation or advancement, priorities, and funding. To provide a Program Manager with credible answers, supporting engineers need to "do their homework" in detail. Virtual prototypes can enhance the communication of the answers to these questions and provide a credible baseline for discussion and analysis. In order to support the program, virtual prototypes must be based on sound engineering and projections of technology growth that are traceable to sources such as programs developing or producing related hardware, expert opinion, field and laboratory tests, and engineering analysis. To produce a high quality accurate model of a technology projection that is credible and useful, each depicted feature of the model must be traceable to one or more of these sources. In this paper, the authors provide examples of virtual prototype accuracy, quality, and handling of technology projections during early technology assessment when many questions remain to be answered. One example projects the size and weight of an electric gun for theater missile defense, and the other example provides a baseline design of a small, heavy metal, waste remediation system for a maintenance facility.

\subsection{Army Electric Gun Example}

During the summer of 1993, the Army successfully tested an electric gun technology that offered potential to perform as a "last ditch" theater ballistic missile defense system (Aden et al. 1997). Virtual prototyping was used to evaluate the readiness of the tested technology to provide a near-term, air-transportable, gun configuration (Schaaf, Churchwell, and Baird 1994). The gun barrel and projectile were known items, but a laboratory power supply that filled a 40 foot semi-trailer had to be projected into a tactical configuration. The resulting solid propellant electrothermal-chemical (SPETC) gun model is assembled from a number of virtual prototypes of individual components of the pulsed power subsystem. The chart in Figure 3 illustrates a worksheet that is a small part of the background work that went into the development of the model. This figure shows

\section{ELECTRICAL POWER:}

Power is related to energy by:

$$
\begin{gathered}
P=\frac{E}{t} \\
P=\text { Power (watts) } \\
E=\text { Energy (joules) } \\
t=\text { Time (seconds) }
\end{gathered}
$$

The SPETC cartridge firing energy requirement is $3.5 \mathrm{MJ}$. $5 \mathrm{MJ}$ is selected for conservative estimates.

$$
\begin{aligned}
& \text { Single Gun Unit } \\
& \text { One gun firing three rounds } \\
& \text { in } 3 \text { seconds } \\
& \text { Gun Fire rate }=1 \mathrm{~Hz} \\
& P_{\text {SHOT }}=\frac{E_{\text {SHOT }}}{1}=\frac{5 \mathrm{MJ}}{1 \text { second }}=5 \mathrm{MW} / \text { charge } \\
& P_{\text {charge }}=5 \mathrm{MW}
\end{aligned}
$$

Three Gun Unit:

Three guns firing a three round burst in 3 seconds (one round each) Gun Fire rate/gun $=.33 \mathrm{~Hz}$ $P_{\text {SHOT }}=\frac{E_{\text {SHOT }}}{t}=\frac{5 \mathrm{MJ}}{3 \text { seconds }}=1.66 \mathrm{MW} /$ charge $P_{\text {charge }}=1.66 \mathrm{MW}$

Electrical power sources for charging the gun firing circuit will add mass and volume to the gun system in proportion to the amount of power required. This mass and volume can be reduced by trades between individual gun fire rates, number of guns per battery, number of rounds per burst, battery fire rate, and time between bursts.

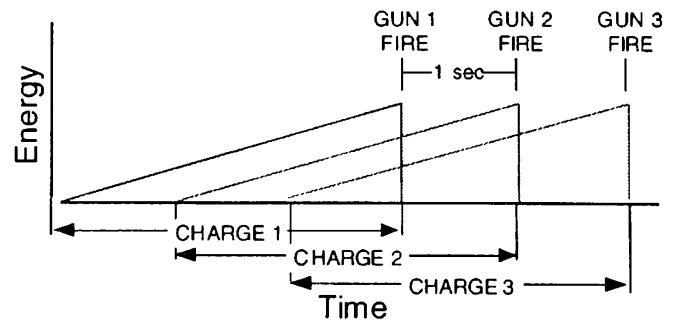

Figure 3: Preliminary Electrothermal Gun Power Sizing Analysis Worksheet 
some of the basic calculations used to show how fire rate requirements drive charging power that in turn drives pulsed power sizing and packaging. This quickly showed that the desired fire rate could not be obtained from a single gun that would meet size and weight constraints, but the fire rate could be met by "ripple firing" three guns that individually met these constraints.

Figure 4 illustrates another worksheet (in briefing viewgraph format) supporting data for the battery subsystem projection that has not been developed outside of the laboratory yet. A literature search and phone calls to academia surfaced research on this battery, and the project engineer provided credible sizing data expected to be achieved in the near future. From this information, a scale 3D computer model of the projected battery pack was constructed to merge with the other subsystems in the computer. This process was repeated for all of the laboratory pulsed power equipment components used in the test. Worksheets on similar models of the more conventional subsystems were also included to explain why some items were shaped the way they were, and how they drove the height and length of the system.

Various mounting configurations of the gun and power system were manipulated and fitted (in the computer monitor) on several different tactical vehicle chassis. Although feasible fits were obtained, each of the self-propelled configurations could not meet weight constraints for C-130 airlift although larger aircraft could accommodate these alternatives. Fitting the gun system on the vehicles showed where chassis and cab modifications would be required to cradle the gun barrel for travel and stay within height constraints. Only the towed version mounted on a semitrailer as shown in Figure 5 met size and weight constraints. This final gun model was assembled from a collection of the various separate subsystem virtual prototypes: gun barrel, mount, pulsed power system, autoloader, and chassis and jacking mechanisms. This configuration uses the lifting bogie from an M2 gun/howitzer carriage to lower the trailer into its firing position. Information and specifications on this mechanism were obtained from the Ordnance Museum at Aberdeen Proving Grounds where one of the authors physically inspected, measured, and photographed the bogie to support modeling. The gun model can be manipulated on the computer screen, and serves as an inexpensive communication tool to generate lively and productive interaction within Integrated Product Teams. This particular towed model was created in about twenty hours by an engineer working on a desktop computer. In contrast, weeks of research before hand were spent gathering data and developing worksheets to ensure there were no misrepresentations of reality in the technology projections.

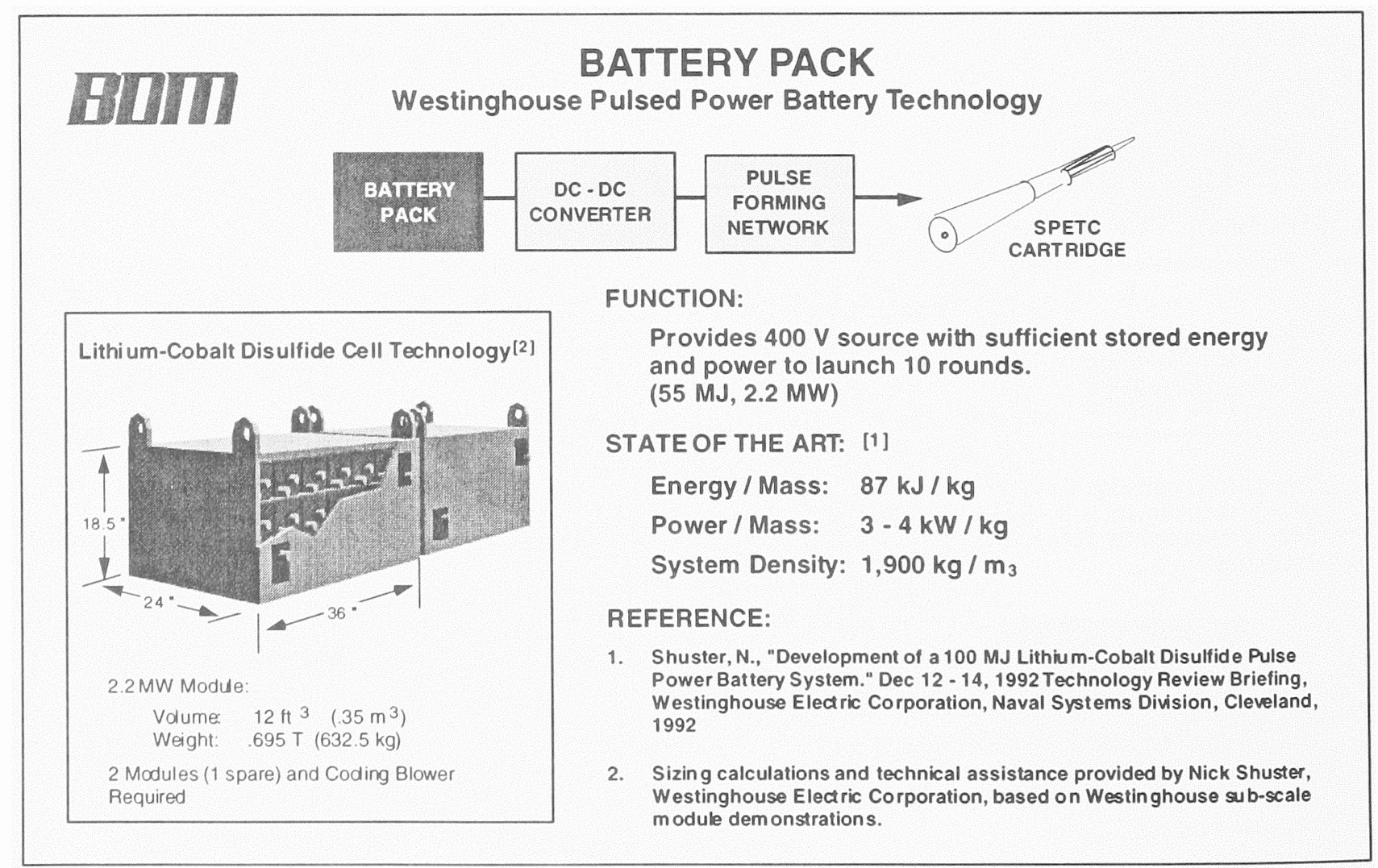

Figure 4: Technology Traceability Worksheet - Battery Pack Sizing 


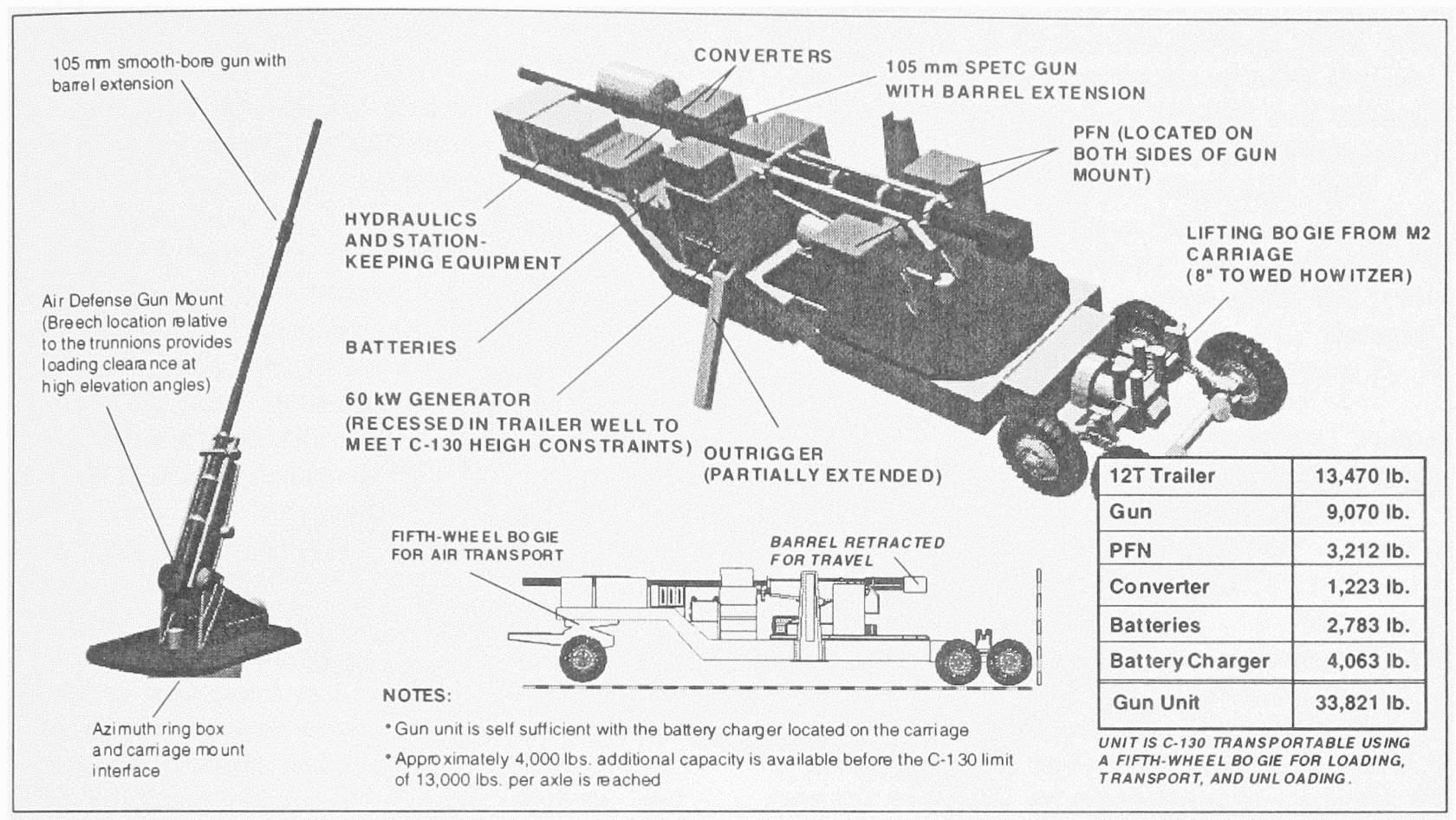

Figure 5: SPETC Gun Concept 3D Model

\subsection{Air Force Waste Remediation Facility Example}

The authors were part of a team formed to adapt a large scale continuous stream waste processing technique (for removing heavy metal waste from water) to smaller scale operations. This waste is generated during Air Force paint removal operations. The smaller scale would allow the Air Force to set up waste remediation facilities at small bases, and avoid the environmental hazards and costs inherent in transporting large volumes of metal laden water to central locations for treatment. Laboratory prototyping and chemical algebra showed that while a small scale continuous stream process was not feasible, batch processing using the same chemical processes was a workable and preferable solution.

Portions of the small batch processing facility were modeled to provide quick answers to human engineering questions. As this analysis progressed, the technique was found to be so useful in responding to broader questions, that the complete facility was modeled from the process and volume flow data that drove storage and processing tank sizes.

In contrast to the electric gun example, the waste facility is designed using proven technology and off-theshelf components. Whereas the gun model emphasized size and weight based on technology projections, the waste facility model emphasizes layout and human factors integration. The facility model did not require as much pre-modeling research as the gun model, and the complete model was developed on a desktop computer in about forty hours. Several weeks were then spent manipulating portions of the model for study.

Virtual prototype development of facilities allows more confident use of catalog components and can reduce the need for custom built structures. The given description and dimensions allow construction of the needed component models which are then inserted into the facility. This "virtual assembly" process points out any areas where catalog components will not meet requirements and custom fabrication is needed. The model then supports development of specifications and drawings for custom components. The drawings, specifications, and instructions can then be sent to the fabricator accompanied by the 3D drawings as a communications aid to ensure that custom or modified items are fabricated correctly .

A "walk through" of the virtual facility on the computer monitor helped identify existing hazards and determine potential failure areas, as well as more advantageous equipment placement. Subsystems can be relocated and moved around in the computer for test fitting and impact analysis as simply as moving toy blocks. Currently needed equipment can initially be placed to take the greatest advantage of facility expansion possibilities, reducing future expansion costs in the future. By studying and manipulating the model, static equipment placement such as support structures, walkways, plumbing, and electrical distribution systems 


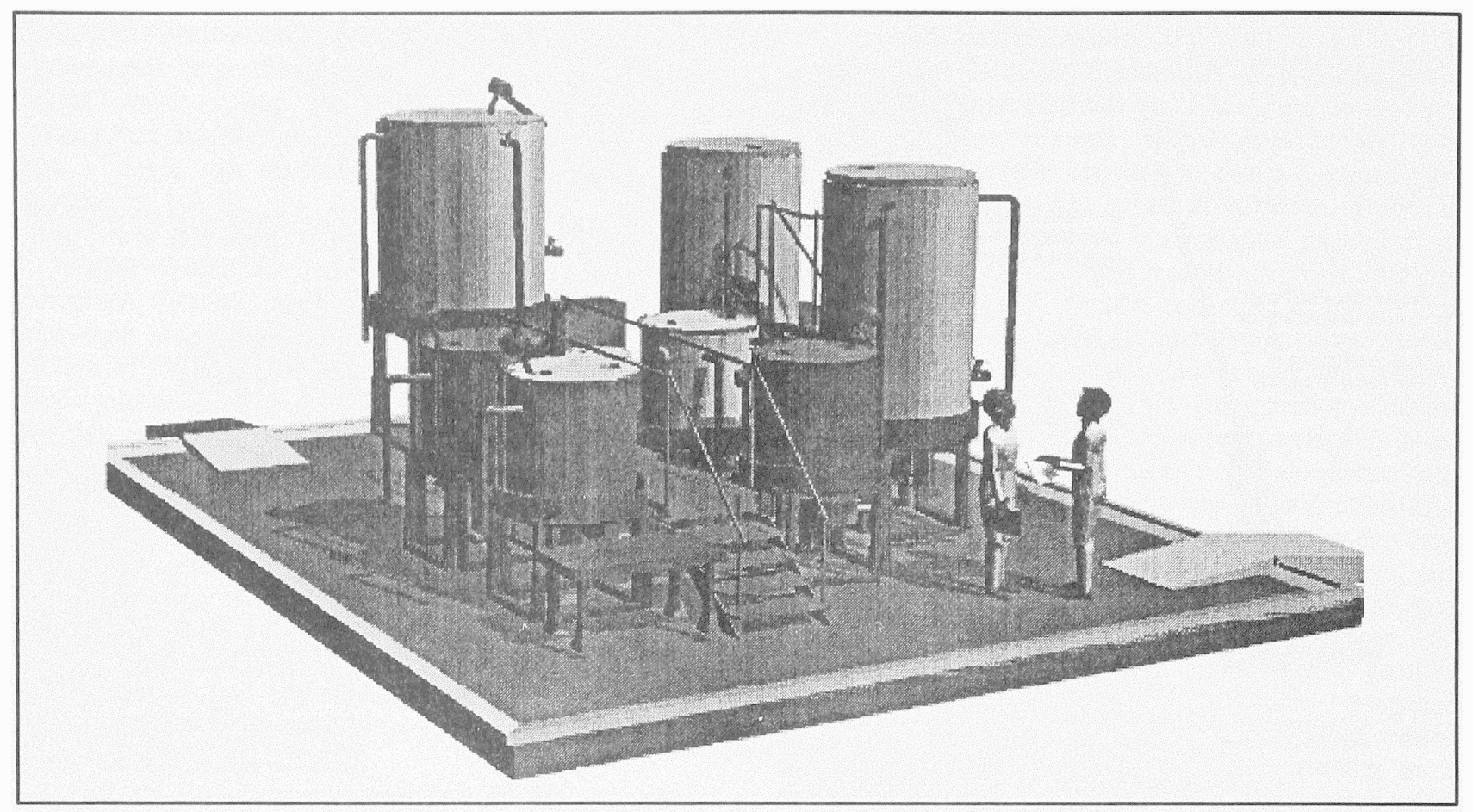

Figure 6: Small Waste Remediation Facility

can be quickly arranged and integrated. The scaled items are simply moved around to provide the proper reach, connection, and clearances. Figure 6 shows a virtual prototype of this industrial waste water remediation facility.

Virtual prototypes also allow clearances for mobile equipment and workspaces to be checked out. Space must be allowed to move canisters of waste and supplies in and out of the facility. This can be done by moving scale models of the ladders and handtrucks within the model. The model also assists the incorporation of Human Factors Engineering. By moving a properly scaled person through the facility, we can ensure that controls and work surfaces can be reached comfortably and safely. This technique was used in placing the stirrer motors and lids on the processing containers. The four middle ( 250 gallon) containers must be manually accessed from above to add reagents, presenting a possible "reach" problem. Virtual prototyping suggested a lid with an off center opening designed to swivel around the stirrer motor bracket.

By using the "object view feature" of most 3D modeling programs, it is possible to see what the operator will see. Simply place the operator model at the various work stations and examine the view from the operators "face." During a final "trip" through the mature model, we identified navigational hazards for the operators, and relocated items to make sure there was nothing to trip on or bump into while moving from the most likely accident sites to the safety equipment. Several pipes were rerouted under the walkway, or on the side of the walkway away from the acid and base canisters and the emergency shower. This technique also resulted in recommending a taller mobile ladder. The ladder was first sized to observe the two 500 gallon containers. An operator's eye view showed that during facility wash down, a clear view of the valve at the bottom of the large (1000 gallon) containers was not possible. A taller ladder with sturdy railing is required to reach the large containers.

Analysis of the facility model suggested additional failure modes which might not otherwise be apparent. The concrete containment pad base is designed to accommodate the contents of all vessels in the system during a catastrophic failure of the plumbing. The model brought to our attention the containment volume used by the concrete ramps required to transport supplies and end products into and out of the facility. These reduced the planned containment volume based on the original design, and the retaining walls around the edge of the pad had to be raised to restore the available confinement volume. In addition, the model pointed out potential problems caused by space constraints that require tanks to be placed close to one another. Failure of one tank support structure could carry it into another tank, toppling both outside the bounds of the concrete containment pad. This was corrected by redesigning the tank support structures and bracing. 


\section{SUMMARY}

Through careful development of details, 3D graphics applications can generate virtual prototypes that provide a useful tool supporting engineering and specialty disciplines analysis for a wide variety of system developments, both early in the concept development phase and later in advanced engineering. Virtual prototyping permits users, designers, and logisticians to visualize two and three dimensional relationships and clearances for joint multi-disciplinary analysis. This early analysis and identification of design issues offers potential to reduce development costs since mistakes will be made in the computer rather than on full-scale prototypes. Due to design maturity, virtual prototypes developed in advanced engineering development will be based on design analysis and known qualities. In contrast, exceptional care must be taken during the technology assessment and advanced concept phases, when details are less certain, to conduct thorough research that produces and supports a credible product based on expert opinion, technology analysis, and traceable assumptions rather than "fantasy." The development of worksheets containing source and rationale information on each virtual component is a disciplined technique that helps to ensure reality is not misrepresented.

\section{REFERENCES}

Aden, T. L., J. L. Brown, V. D. Churchwell, P. Dewar, A. Juhasz, Z. Kaplan, and J. Williams. 1997. Summary of the Hypervelocity Weapon System Field Experiment, IEEE Transactions on Magnetics, 33(1):636-641

Garcia, A. B., R. P. Gocke, and N. P. Johnson. 1993. Virtual Prototyping, Concept to Production, Defense System Management College, Ft. Belvoir, VA.

Murie, Michael D. 1996. Windows' Third Dimension, New Media, January, 62-69

Piplani, L. K., J. G. Mercer, and R. O. Roup.1994. System Acquisition Manager's Guide for the Use of Models and Simulation, Defense System Management College, Ft. Belvoir, VA.

Potter, Caren D. 1996. Sorting Through the Bargain Bin of 3D CAD, Computer Graphics World, January, 23-25
Schaaf, J. C., V. D. Churchwell, and S. D. Baird. 1994. Size and Weight Development of Solid Propellant Electrothermal Chemical (SPETC) Gun Concepts, Poster Session 103F for the 7th Electromagnetic Launcher Symposium, San Diego, CA, April.

Schaaf, J. C., F. L. Thompson,, and V. D. Churchwell. 1994. Virtual Prototyping with Low-end 3D Graphics Programs, Proceedings of the 1994 Winter Simulation Conference, Orlando, FL, December, 798-804, ed: J. D. Trew, S. Manivannan, D. A. Sadowski, and A. F. Seila, IEEE

Stewart \& Stevenson Services, Inc. 1993. Tactical Vehicle Systems Division, data sheets and drawings for the 5-Ton MTV chassis series, Sealy, TX.

\section{AUTHOR BIOGRAPHIES}

JAMES C. SCHAAF, JR. is a Senior Systems Engineer in the Technology Applications Group at BDM International in Huntsville. He became interested in the application of low-end 3D graphics programs to meet the demanding art requirements of a research and development client, and has gradually evolved the application from an art generator to an engineering tool supporting concept development. His most recent application of virtual prototyping includes the design of a magnetic levitation test fixture. The model and 3D renderings proved to be a significant communication aid in developing the final design with a fabrication shop. $\mathrm{He}$ is a member of IEEE, ADPA, AUSA, and SOLE.

FAYE LYNN THOMPSON is a Systems Analyst in the Strategic Applications Directorate of BDM International in Huntsville. Her research interests involve combining formal training in physics, art and optics to enhance technical understanding among various technology teams, and increase the appreciation of nontechnical subjects among scientist and engineers. Her most recent application of virtual prototyping involved the design and layout of a heavy metal waste remediation facility. Manipulation and analysis of this model on the computer screen identified numerous problems and improvements in facility layout and operator safety features. She is a member of Sigma Pi Sigma, SPIE and participates in a local photographic society as well as working in stained glass. 